\title{
Distributive Radiation and Transfer Characterization Based on the PEEC Method
}

\author{
Ying S. Cao, Student Member, IEEE, Li Jun Jiang, Senior Member, IEEE, and Albert E. Ruehli, Life Fellow, IEEE
}

\begin{abstract}
Power radiation and radiated coupling among different structures represent an important area for research study. The partial element equivalent circuit method (PEEC) is a powerful technique which bridges the gap between the electromagnetic and circuit theories. In this paper, we develop a new approach for PEEC to calculate the distributive power radiation and couplings. With this approach, formulations for both the radiated power and the transferred power can be evaluated. Further, the physical meanings of the power results are interpreted according to the conservation of energy law. We use electric dipoles, magnetic dipoles and patch antennas as well as coupled microstrip lines to benchmark the method. The approach can also be applied to EMC/EMI and other power dissipation computations.
\end{abstract}

Index Terms-Energy conservation, partial element equivalent circuit method (PEEC), radiated power, transferred power.

\section{INTRODUCTION}

$\mathbf{T}$ HE rising speed and numbers of electronic devices made today as well as the use of new artificial materials [1] make EMC/electromagnetic interference (EMI) issues of ever increasing importance. With the increasing density of devices and bandwidth of the signal channel, the complexity of ICs and related interconnects are becoming bottlenecks for many high performance systems. Electronic design automation software is indispensable for successful designs. However, modeling of high speed signals in a complex environment is not a trivial task. To make a tradeoff between speed and accuracy, many empirical or theoretical approximations have to be used at different stages of designs.

With the improvement of devices, computational methods and commercial softwares also have to be improved. Various approaches such as the method of moments [4], finite element method [3], and finite difference methods [2] are developed based on numerical methods. For example, full-wave solvers have been developed by using the augmented electric field integral equation (EFIE) [5] aiming at a better solution of the low frequency regime. These computational methods or softwares focus on characterizing ports, such as the matching conditions or insertion loss. Results are efficiency, radiation patterns and

Manuscript received October 6, 2014; revised December 2, 2014; accepted December 7, 2014. This work was supported in part by Hong Kong RGC GRF 712612, GRF 711511, NSFC 61271158, US AR120018 contracted through UTAR and Hong Kong UGC AoE/P04/08.

Y. S. Cao and L. J. Jiang are with the Department of Electrical and Electronic Engineering, University of Hong Kong, Hong Kong (e-mail: caoying@eee.hku. hk; jianglj@hku.hk).

A. E. Ruehli is with the UMRI/MST EMC Laboratory, Missouri University of Science and Technology, Rolla, MO 65409 USA (e-mail: albert.ruehli@ gmail.com).

Color versions of one or more of the figures in this paper are available online at http://ieeexplore.ieee.org.

Digital Object Identifier 10.1109/TEMC.2014.2382176 also current distributions, etc. However, these approaches blend together internal physical phenomena. Issues like radiation and coupling between different components are not transparent to users in these methods.

The partial element equivalent circuit (PEEC) method, which was proposed in the 1970s [6]-[9], is based on the EFIE with potentials and currents as unknowns suitable for circuit models for electromagnetic problems. The approach turns the field into a circuit problem. A large category of PEEC applications are for static or quasi-static problems. Under these conditions, partial elements in PEEC are real if the retardation is approximated or ignored. This obviously is not suitable for higher frequencies. For high frequency full-wave solutions, the partial elements should include full dynamic Green's functions to represent radiation effects. Hence, we employ the full-wave PEEC method in this study for power radiation and energy transfer problems.

In [10], [11], an approach has been proposed to use the PEEC method to decompose the radiation part and evaluate the distributive contributions for different structures. The approach includes retardation and extracts all real power results from a phasor power representation. The radiation and power transfer are separated by using partial elements. It clearly determines the power composition of radiators. However, the representations of power transfer and radiation in [10] have some issues. Another recent study [13] which evaluates the radiated power for PCB is based on the port network parameters. The radiated power is calculated using incident port voltages and $S$-parameters.

In our approach, the geometrical current distributions and the electric field are found. In [14], a new radiation calculation method was proposed using the retardation and the reorganization of the partial components. It predicts the Hertzian dipole very well. However, it is difficult to be used for general large structures. In our study, we extend the PEEC method to distributive radiation and power transfer analysis such that details like the radiation and contributions to couplings from each segment of the radiator can be singled out. The differences between this study and [10] is that we not only derive the power formulations in terms of self- and mutual terms according to the energy conservation law, but also provide a clear physical interpretation of both the radiated and transferred power results. We analyze the coupling between radiated objects as well as the coupling and radiation of different parts for the same radiator. This study can be applied to EMI optimization, noise coupling reduction, and other IC and PCB applications.

The paper is organized as follows: Section II will give a brief introduction on the old and new power radiation and transfer formulations; Section III demonstrates the radiated and transferred power based on the energy conservation law; Section IV investigates several benchmarks to verify the proposed idea. 


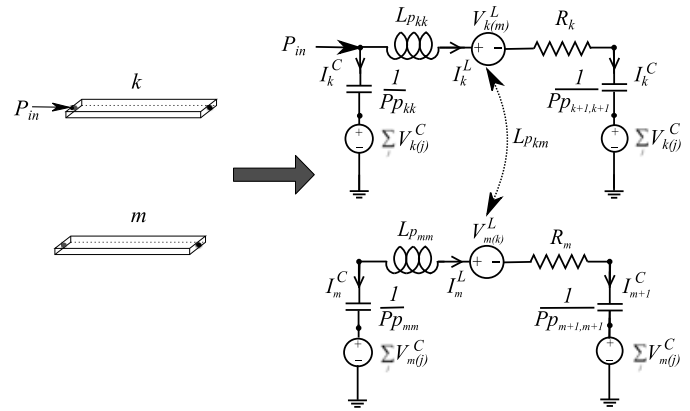

Fig. 1. Power into two cells and their equivalent PEEC model. $P_{\text {in }}$ is the total input power into the two cells, in which a sinusoidal source is applied.

Conclusions and discussions are presented at the end of this paper.

\section{THEORETICAL BACKGROUND}

Fig. 1 depicts a representative PEEC circuit model for cell $k$ and cell $m$ in the MLA form as is the case in [10]. There are controlled voltage sources on inductive-coupled branches. They are

$$
\begin{aligned}
& V_{k(m)}^{L}=j \omega L p_{k m} I_{m}^{L} e^{-j K d_{k m}} \\
& V_{m(k)}^{L}=j \omega L p_{k m} I_{k}^{L} e^{-j K d_{k m}}
\end{aligned}
$$

where $d_{k m}$ is the center-to-center distance between two inductive cells $k$ and $m . L p_{k m}$ is the partial mutual inductance between cell $k$ and $m$, and is a pure real value here. $K$ here is the wave number.

For two capacitive branches $k$ and $m$ the controlled voltage sources are

$$
\begin{aligned}
V_{k(m)}^{C} & =\frac{P p_{k m}}{j \omega} I_{m}^{C} e^{-j K d_{k m}} \\
V_{m(k)}^{C} & =\frac{P p_{k m}}{j \omega} I_{k}^{C} e^{-j K d_{k m}}
\end{aligned}
$$

where $d_{k m}$ is the central distance between two capacitive cells $k$ and $m . I_{m}^{C}$ and $I_{k}^{C}$ are currents through the $m$ th and $k$ th capacitive branch, respectively. $P p_{k m}$ is the partial coefficient of potential between cell $k$ and $m$, which is a real number in this formulation. Details of the formulation are given in [10]. We note that the midfrequency approximation of the retardation does not include the contribution of the self-terms to the radiation. We also note that the self-term corresponds to a Hertzian dipole [14]. It accurately presents the retardation.

This issue is solved here by accurately using the full wave Green's function in the integration kernel. This results in complex partial inductances and partial coefficients of potential. Let $L p_{k m}$ represent the complex inductance, and $P p_{k i}$ represent the complex coefficient of potential. The branch currents $I_{m}$ and $I_{k}$ are also complex currents in circuit $m$ and $k$. Then

$$
L p_{k m}=\frac{\mu}{4 \pi a_{k} a_{m}} \int_{a_{k}} \int_{a_{m}^{\prime}} \int_{l_{k}} \int_{l_{m}^{\prime}} \frac{e^{-j K\left|\mathbf{r}-\mathbf{r}^{\prime}\right|}}{\left|\mathbf{r}-\mathbf{r}^{\prime}\right|} d a_{k} d a_{m}^{\prime} d l_{k} d l_{m}^{\prime}
$$

$$
P p_{k i}=\frac{1}{4 \pi S_{i} S_{k} \varepsilon} \int_{S_{k}} \int_{S_{i}^{\prime}} \frac{e^{-j K\left|\mathbf{r}-\mathbf{r}^{\prime}\right|}}{\left|\mathbf{r}-\mathbf{r}^{\prime}\right|} d S_{k} d S_{i}^{\prime}
$$

where again $K$ is the wave number. Then (1) can be rewritten as

$$
\begin{aligned}
& V_{k(m)}^{L}=j \omega L p_{k m} I_{m}^{L} \\
& V_{m(k)}^{L}=j \omega L p_{k m} I_{k}^{L} .
\end{aligned}
$$

Of course, the self-terms are obtained if we set $k=m$ in aforementioned equations to get the complex partial selfinductances and partial self-coefficients of potential.

After mathematical manipulations, the transferred and radiated power can be represented by partial elements and current distributions on meshes, which are

$$
\begin{aligned}
P_{m(k)}^{L, r} & =\frac{1}{2} \operatorname{Re}\left(I_{k}^{L} I_{m}^{L *}\right) \cdot \operatorname{Re}\left(j \omega L p_{k m}\right) \\
P_{m(k)}^{L, t} & =-\frac{1}{2} \operatorname{Im}\left(I_{k}^{L} I_{m}^{L *}\right) \cdot \operatorname{Im}\left(j \omega L p_{k m}\right) \\
P_{m(k)}^{C, r} & =\frac{1}{2} \operatorname{Re}\left(I_{k}^{C} I_{m}^{C *}\right) \cdot \operatorname{Re}\left(\frac{P p_{k m}}{j \omega}\right) \\
P_{m(k)}^{C, t} & =-\frac{1}{2} \operatorname{Im}\left(I_{k}^{C} I_{m}^{C *}\right) \cdot \operatorname{Im}\left(\frac{P p_{k m}}{j \omega}\right)
\end{aligned}
$$

where $P_{m(k)}^{L, r}$ and $P_{m(k)}^{L, t}$ are the inductive coupled radiated and transferred power for cell $m$ by coupling with $k$, respectively. $P_{m(k)}^{C, r}$ and $P_{m(k)}^{C, t}$ are the capacitive radiated and transferred power for cell $m$ by coupling with $k$, respectively. And the total radiated and transferred power is the sum of both inductive and capacitive effects. Further details can be referred to [12]. Therefore

$$
\begin{aligned}
& P_{m(k)}^{r}=P_{m(k)}^{L, r}+P_{m(k)}^{C, r} \\
& P_{m(k)}^{t}=P_{m(k)}^{L, t}+P_{m(k)}^{C, t} .
\end{aligned}
$$

\section{ENERGY CONSERVATION}

\section{A. Radiated Power Analysis}

1) Two Cells: For the case with only two PEEC loops as shown in Fig. 1, the total power for loops $k$ and $m$ is

$$
\begin{aligned}
P_{\mathrm{in}}= & \frac{1}{2} \operatorname{Re}\left(\left(R_{k}+j \omega L p_{k k}\right) I_{k}^{L 2}+\left(R_{m}+j \omega L p_{\mathrm{mm}}\right) I_{m}^{L 2}\right. \\
& \left.+V_{k(m)}^{L} I_{k}^{L *}+V_{m(k)}^{L} I_{m}^{L *}\right) \\
& +\frac{1}{2} \operatorname{Re}\left(\frac{P p_{k k}}{j \omega} I_{k}^{C 2}+\frac{P p_{k+1, k+1}}{j \omega} I_{k+1}^{C 2}\right. \\
& \left.+\frac{P p_{\mathrm{mm}}}{j \omega} I_{m}^{C 2}+\frac{P p_{m+1, m+1}}{j \omega} I_{m+1}^{C 2}\right) \\
& +\frac{1}{2} \operatorname{Re}\left(\sum_{j \neq k} V_{k(j)}^{C} I_{k}^{C *}+\sum_{j \neq k+1} V_{k+1(j)}^{C} I_{k+1}^{C *}\right. \\
& \left.+\sum_{j \neq m} V_{m(j)}^{C} I_{m}^{C *}+\sum_{j \neq m+1} V_{m+1(j)}^{C} I_{m+1}^{C *}\right) .
\end{aligned}
$$




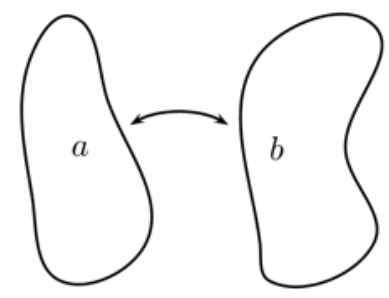

Fig. 2. Geometry of two general coupled structures.

From the equation, the input power can be separated into three parts:

1) Ohmic power $\frac{1}{2}\left(R_{k} I_{k}^{L 2}+R_{m} I_{m}^{L 2}\right)$.

2) Self-radiated power, marked as $P_{k(k)}^{r^{\prime}}$ and $P_{m(m)}^{r^{\prime}}$ to distinguish them from the power defined in (5).

$$
\begin{aligned}
P_{k(k)}^{r^{\prime}}= & \frac{1}{2} \operatorname{Re}\left(j w L p_{k k} I_{k}^{L 2}\right. \\
& +\frac{P p_{k k}}{j \omega} I_{k}^{C 2}+\frac{P p_{k+1, k+1}}{j \omega} I_{k+1}^{C 2} \\
& \left.+V_{k(k+1)}^{C} I_{k}^{C *}+V_{k+1(k)}^{C} I_{k+1}^{C *}\right) \\
P_{m(m)}^{r^{\prime}}= & \frac{1}{2} \operatorname{Re}\left(j w L p_{\mathrm{mm}} I_{m}^{L 2}\right. \\
& +\frac{P p_{\mathrm{mm}}}{j \omega} I_{m}^{C 2}+\frac{P p_{m+1, m+1}}{j \omega} I_{m+1}^{C 2} \\
& \left.+V_{m(m+1)}^{C} I_{m}^{C *}+V_{m+1(m)}^{C} I_{m+1}^{C *}\right) .
\end{aligned}
$$

The self-radiated power has two parts: inductive selfradiation and capacitive self-radiation.

3) The radiated power due to mutual coupling also has two parts $P_{m(k)}^{r^{\prime}}$ and $P_{k(m)}^{r^{\prime}}$, to distinguish from the power defined in (5), and they are

$$
\begin{aligned}
P_{m(k)}^{r^{\prime}}= & \frac{1}{2} \operatorname{Re}\left(V_{k(m)}^{L} I_{m}^{L *}+\sum_{j \notin \mathrm{cell} m} V_{m(j)}^{C} I_{m}^{C *}\right. \\
& \left.+\sum_{j \notin \operatorname{cell} m} V_{m+1(j)}^{C} I_{m+1}^{C *}\right) \\
P_{k(m)}^{r^{\prime}}= & \frac{1}{2} \operatorname{Re}\left(V_{k(m)}^{L} I_{k}^{L *}+\sum_{j \notin \mathrm{cell} k} V_{k(j)}^{C} I_{k}^{C *}\right. \\
& \left.+\sum_{j \notin \mathrm{cell} k} V_{k+1(j)}^{C} I_{k+1}^{C *}\right) .
\end{aligned}
$$

The radiated power due to mutual couplings also has two parts: the mutual inductive power and mutual capacitive power.

2) General Structure Case: For the case where we have two coupled arbitrary structures, each structure is discretized into small mesh cells using the PEEC scheme. We can get the current distribution on each cell by solving the lumped circuit model. In Fig. 2, the radiated power can be simplified in a vector-matrixvector product form.

$$
P_{a(a)}^{r^{\prime}}=\frac{1}{2} \operatorname{Re}\left(\mathbf{I}_{a}^{L^{\dagger}} \overline{\mathbf{R}}_{L}^{a a} \mathbf{I}_{a}^{L}+\mathbf{I}_{a}^{C \dagger} \overline{\mathbf{R}}_{C}^{a a} \mathbf{I}_{a}^{C}\right)
$$

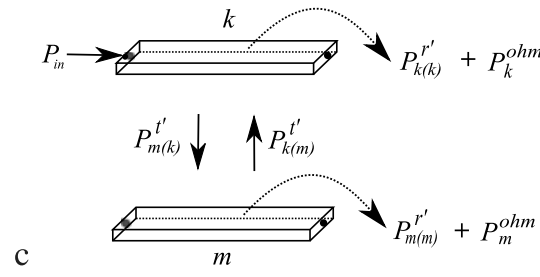

Fig. 3. Geometry of two arbitrary coupled cells.

$$
\begin{aligned}
P_{b(b)}^{r^{\prime}} & =\frac{1}{2} \operatorname{Re}\left(\mathbf{I}_{b}^{L^{\dagger}} \overline{\mathbf{R}}_{L}^{b b} \mathbf{I}_{b}^{L}+\mathbf{I}_{b}^{C \dagger} \overline{\mathbf{R}}_{C}^{b b} \mathbf{I}_{b}^{C}\right) \\
P_{a(b)}^{r^{\prime}} & =\frac{1}{2} \operatorname{Re}\left(\mathbf{I}_{a}^{L^{\dagger}} \overline{\mathbf{R}}_{L}^{a b} \mathbf{I}_{b}^{L}+\mathbf{I}_{a}^{C \dagger} \overline{\mathbf{R}}_{C}^{a b} \mathbf{I}_{b}^{C}\right) \\
P_{b(a)}^{r^{\prime}} & =\frac{1}{2} \operatorname{Re}\left(\mathbf{I}_{b}^{L^{\dagger}} \overline{\mathbf{R}}_{L}^{a b} \mathbf{I}_{a}^{L}+\mathbf{I}_{b}^{C \dagger} \overline{\mathbf{R}}_{C}^{a b} \mathbf{I}_{a}^{C}\right)
\end{aligned}
$$

in which the superscript ' $\dagger$ ' means the conjugate transpose of a vector. $\mathbf{I}_{a}^{L}$ and $\mathbf{I}_{b}^{L}$ are vectors of current distribution of inductive branches on $a$ and on $b$, respectively. $\mathbf{I}_{a}^{C}$ and $\mathbf{I}_{b}^{C}$ are current distribution vectors for capacitive branches on $a$ and $b$, respectively; $\overline{\mathbf{R}}_{L}^{a b}$ is an $N_{L_{a}} \times N_{L_{b}}$ matrix in which all elements are inductive coupling impedances ( $N_{L_{a}}$ and $N_{L_{b}}$ are the number of inductive cells on $a$ and $b$, separately. $\left.\overline{\mathbf{R}}_{L(m k)}^{a b}=j \omega L p_{m k}\right)$, and the $\overline{\mathbf{R}}_{C}^{a b}$ is an $N_{C_{a}} \times N_{C_{b}}$ matrix whose elements correspond to capacitive coupling ( $N_{C_{a}}$ and $N_{C_{b}}$ are number of capacitive cells on $a$ and $b$, respectively. $\left.\overline{\mathbf{R}}_{C(m k)}^{a b}=\frac{P p_{m k}}{j \omega}\right)$.

For the traditional power analysis, the radiated power based on mutual coupling is not transparent from the formulation. In this study, the power for mutual couplings is obtained and its physical significance is also figured out. It shows how each part of the structure contributes to the total radiation. In (10), $P_{a(b)}^{r^{\prime}}$ can be positive or negative. If it is positive, it means coupled radiated power of $a$ due to coupling with $b$ enhances the total radiation of the structure. If it is negative, it means the coupled-radiated power of $a$ by coupling with $b$ decreases the total radiation of the system. This provides a unique analysis for both radiation optimization and noise reduction.

\section{B. Analysis of Transferred Power}

With the proposed radiated power definition, we can carefully derive the representation of the transferred power. Similar to the radiated power, we will have mutually transferred power as well as self-transferred power. But here we care more about the mutually transferred power since it represents how much power is transferred from one part to another.

In Fig. 3, only cell $k$ receives injected power $P_{\text {in }}$. The power consumption on $m$ contains ohmic power and radiated power, which are $P_{\mathrm{mm}}^{r^{\prime}}$ and $P_{m}^{\mathrm{ohm}}$. According to energy conservation, energy can be neither created nor destroyed. It can only be converted from one form to another. Since $m$ is passive, there is no source on it, all of its power comes from $k$. This is called the transferred power from $k$ to $m$, i.e., $P_{m(k)}^{t^{\prime}}$. The transferred power from $k$ to $m$ shall be equal to power consumption on $m$. 
Hence, we have

$$
P_{m(k)}^{t^{\prime}}=P_{m(m)}^{r^{\prime}}+P_{m}^{\mathrm{ohm}} .
$$

The remaining question is the computation of $P_{m(k)}^{t^{\prime}}$. The right hand side of (7) can be separated into three parts. The radiated power based on the mutual coupling can be moved to the left hand side of the equation. Hence, the equation can be rewritten as

$$
\begin{aligned}
& P_{\text {in }}-\frac{1}{2} \operatorname{Re}\left(\sum_{i \notin \text { cell } k} V_{k(i)}^{L} I_{m}^{L *}\right. \\
& +\sum_{j \notin \text { cell } k} V_{k(j)}^{C} I_{k}^{C *}+\sum_{j \notin \text { cell } k} V_{k+1(j)}^{C} I_{k+1}^{C *} \\
& +\sum_{i \notin \text { cell } m} V_{m(i)}^{L} I_{m}^{L *} \\
& \left.+\sum_{j \notin \operatorname{cell} m} V_{m(j)}^{C} I_{m}^{C *}+\sum_{j \notin \operatorname{cell} m} V_{m+1(j)}^{C} I_{m+1}^{C *}\right) \\
& =\mathrm{P}_{k}^{\mathrm{ohm}}+P_{m}^{\mathrm{ohm}}+\mathrm{P}_{k(k)}^{r^{\prime}}+P_{m(m)}^{r^{\prime}} .
\end{aligned}
$$

By comparing (12) with (11), we can safely come to the conclusion that

$$
\begin{aligned}
P_{m(k)}^{t^{\prime}}= & -\frac{1}{2} \operatorname{Re}\left(\sum_{i \notin \text { cell } m} V_{m(i)}^{L} I_{m}^{L *}+\sum_{j \notin \operatorname{cell} m} V_{m(j)}^{C} I_{m}^{C *}\right. \\
& \left.+\sum_{j \notin \text { cell } m} V_{m+1(j)}^{C} I_{m+1}^{C *}\right) .
\end{aligned}
$$

Below we will provide several examples to benchmark this equation. If the transferred power $P_{m(k)}^{t^{\prime}}$ calculated by (13) is the same to $P_{m(m)}^{r^{\prime}}+P_{m}^{\mathrm{ohm}}$ calculated by (10), then we can say that the formulation of transferred power is correct.

\section{Physical Interpretation of Power Transfer and Radiation}

As shown in (3) the partial elements (partial inductances and partial coeffcients of potential) are represented by the Green's function. For low frequencies or conductors which are physically close, the Green's function can be expanded in a Taylor series. According to (3), the partial elements are complex.

The different parts of the series expansion of the partial elements make different contributions to the power transfer as well as to the radiation.

$$
\begin{aligned}
\frac{e^{-j K R}}{R}= & \frac{1}{R} \sum_{n=0}^{\infty} \frac{(-j K R)^{n}}{n !}=\frac{1}{R}(1+(-j K R) \\
& \left.+\frac{(-j K R)^{2}}{2 !}+\frac{(-j K R)^{3}}{3 !}+\ldots\right) .
\end{aligned}
$$

1) Inductive Branch: The inductive impedance is obtained if we multiply (14) by $j \omega$. The first term in (14), which is $\frac{1}{R}$, corresponds to an inductor. The second term $\frac{-j K R}{R}$, corresponds to a positive resistor. Further, the fourth term $\frac{(-j K R)^{3}}{3 ! \cdot R}$ actually corresponds to a negative resistor. The combination of the positive resistor and the negative resistor results in a physical resistor with a positive value. The third term $\frac{(-j K R)^{2}}{2 ! \cdot R}$ corresponds to a

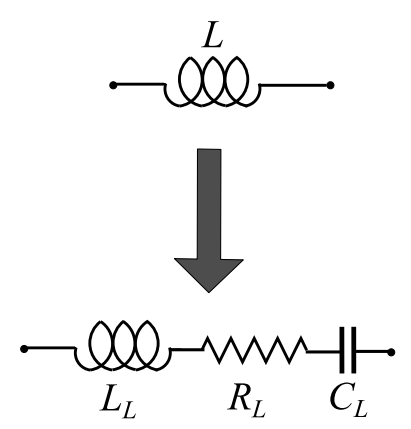

Fig. 4. Physical process on inductive branch. During this process, a numerical complex inductor is turned into a series of a physical inductor $L_{L}$, a positivevalued resistor $R_{L}$ and a capacitor $C_{L}$, among which only $R_{L}$ contributes to radiation.

capacitor. These four elements are obtained from the first four terms of the Taylor series. We assume that the maximum frequency is low enough or that the distance between the elements is small enough that such that $K R \ll 1$. The equivalent circuit for the partial inductance is shown in Fig. 4.

After certain mathematical manipulations, $L_{L}, R_{L}$ and $C_{L}$ can be represented as follows:

$$
\begin{aligned}
L_{L} \propto \sum_{n} \frac{(K R)^{n}}{R \cdot n !}, n & =0,4,8 \ldots \\
R_{L} \propto \sum_{n}(-1)^{\frac{n-1}{2}} \cdot \frac{\omega \cdot(K R)^{n}}{R \cdot n !}, n & =1,3,5 \ldots \\
C_{L} \propto \sum_{n} \frac{R n !}{K^{n+2} R^{n} c^{2}}, n & =2,6,10 \ldots
\end{aligned}
$$

in which $\omega$ is the angular frequency, $K$ is wave number and $c$ is the velocity of light in air. For an accurate Taylor series expansion it is required that $K R \ll 1$. Considering (15), we can see that $L_{L}$ will increase for higher frequencies and $C_{L}$ will decrease. For $R_{L}$, it has positive and negative parts according to (15b). Since $K R \ll 1$, the first term of $R_{L}$ dominates. Moreover, $R_{L}$ is always positive, and it can be proved that $R_{L}$ is the only part in these three components which contributes to radiation. And the radiated power by $R_{L}$ is always positive.

2) Capacitive Branch: Similarly, on the capacitive branch, if (14) is divided by $j \omega$, the capacitive impedance will be obtained, as shown in Fig. 5.

$$
\begin{array}{r}
C_{C} \propto \sum_{n} \frac{(K R)^{n}}{R \cdot n !}, n=0,4,8 \ldots \\
R_{C} \propto \sum_{n}(-1)^{\frac{n+1}{2}} \cdot \frac{(K R)^{n}}{\omega R \cdot n !}, n=1,3,5 \ldots \\
L_{C} \propto \sum_{n} \frac{K^{n-2} R^{n}}{n ! R c^{2}}, n=2,6,10 \ldots
\end{array}
$$

in which $\omega$ is the angular frequency, $K$ is wave number and $c$ is the velocity of light in air. According to requirements for Taylor expansion, $K R \ll 1$. Looking back to (16), we can see that $C_{C}$ will become larger when frequency goes higher. The 


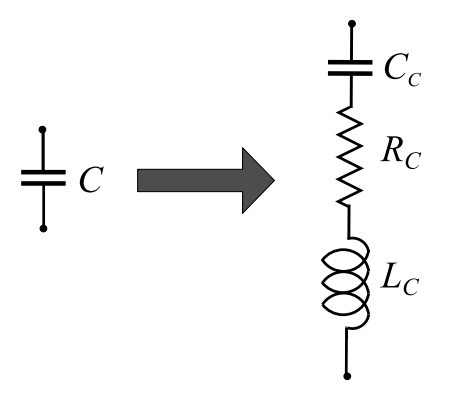

Fig. 5. Physical process on capacitive branch. During this process, a numerical complex capacitor is turned into a series of a physical capacitor $C_{C}$, a resistor $R_{C}$ and an inductor $L_{C}$, among which only $R_{C}$ contributes to radiation.

first term of $L_{C}$ is only proportional to $r$, and does not depend on frequency. The following terms of $L_{C}$ will increase when frequency goes higher. For $R_{C}$, it has positive and negative parts according to (16 b), and the sum of these terms is negative. Since $K R \ll 1$, the first term of $R_{C}$ dominates. Moreover, $R_{C}$ is always negative, and it can be proved that $R_{C}$ is the only part in these three components which contributes to the radiation. And the radiated power by $R_{L}$ is always negative.

To sum up the radiation process from both inductive and capacitive effects, only $R_{L}$ and $R_{C}$ contribute to it. This is because power on these resistors are nearly pure real. $L_{L}, L_{C}$ part and $C_{L}, C_{C}$ part correspond to stored magnetic power and electric power, respectively, and power for the inductors and capacitors are almost pure imaginary. This agrees with the Poynting theorem (PT), which states that the real part of power corresponds to radiation loss and ohmic loss, and imaginary part is for the stored power (magnetic power and electric power).

For the transferred power, $R_{L}, R_{C}, L_{L}, L_{C}, C_{L}$ and $C_{C}$ part all have contributions. They can enhance or decrease the power transfer process due to different frequencies or positions.

\section{NUMERICAL VERIFICATION}

\section{A. Benchmark Procedure}

In order to validate the proposed formulations for the radiated and transferred power, we use two coupled structures represented by $a$ and $b$. Here, $a$ is excited with a source while $b$ has a passive load.

We will do the experiments in three steps:

1) Verify the self-radiated power. We use (10a) or (10b) to calculate radiated and ohmic power of $a$ and $b$, respectively. This is compared with power results using the PT or softwares such as ADS, HFSS, etc.

2) Verify the transferred power. Also, we calculate $P_{b(a)}^{t^{\prime}}$ by (13) and compare it with the summation of radiated power and ohmic power in stage $(a)$. If $P_{b(a)}^{t^{\prime}}=P_{b(b)}^{r^{\prime}}+$ $P_{b}^{\mathrm{ohm}}$, the formulation of transferred power is proven to be correct.

3) Verify the radiated power due to mutual couplings.

Traditional method does not lead to the determination of the mutual-radiated power. We need to compare the total-radiated power of the system $P^{r^{\prime}}$ calculated by (10a) or (10b), with the

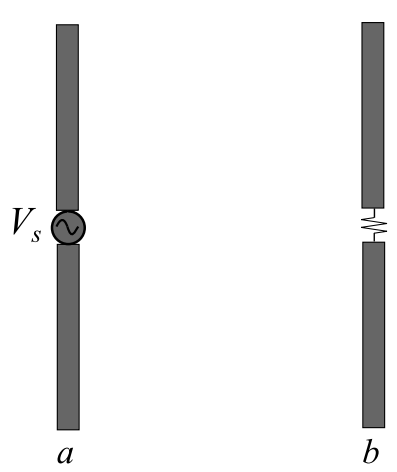

Fig. 6. Geometry of two coupled dipoles.

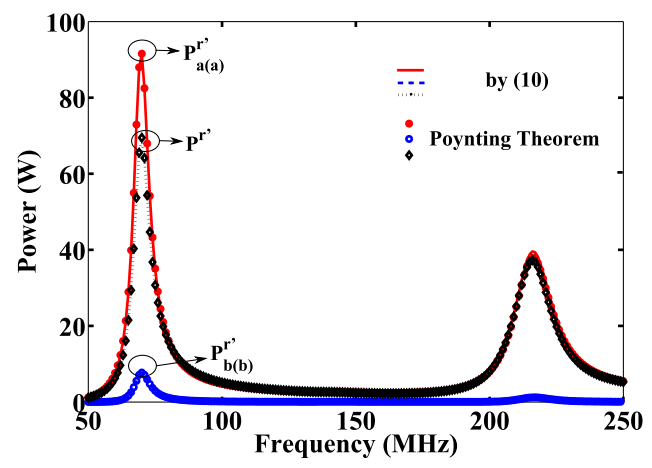

Fig. 7. Radiated power of two dipoles calculated by (10) compared with the PT. $P^{r^{\prime}}$ is the total-radiated power of the system. $P_{a(a)}^{r^{\prime}}$ and $P_{b(b)}^{r^{\prime}}$ are selfradiated power by "a" and "b," respectively.

sum of the self- and mutual-radiated power of the radiators in the system, say $P_{a(a)}^{r^{\prime}}, P_{b(b)}^{r^{\prime}}$ calculated by (10a) and (10b), $P_{b(a)}^{r^{\prime}}$ and $P_{a(b)}^{r^{\prime}}$ which are calculated by (10c) and (10d). According to stage (a), self-radiated power can be verified by the PT. If $P^{r^{\prime}}=$ $P_{a(a)}^{r^{\prime}}+P_{b(b)}^{r^{\prime}}+P_{a(b)}^{r^{\prime}}+P_{b(a)}^{r^{\prime}}$, we conclude that formulations of mutual-radiated power are also correct.

\section{B. Two Dipoles}

Two lossless, zero-thickness dipoles are both $2 \mathrm{~m}$ long and $1 \mathrm{~cm}$ wide which are placed to be parallel to each other. The center to center distance between them is $1 \mathrm{~m}$. The receiver is loaded with a $100-\Omega$ resistor while the transmitter is excited by a lumped sinusoidal voltage source with the amplitude $V_{S}=100$ V. The geometry is illustrated in Fig. 6. From Fig. 7, we can see that self-radiated power from (10) matches very well with what we get from the PT. And the radiated power results reach maximum at around 75 and $225 \mathrm{MHz}$ which are the resonant frequencies of the dipoles. Fig. 8 illustrates that transferred power matches very well with power consumption on $b$, which means that the transferred power formulation derived from the energy conservation law is correct. And the transferred power results reach maximum at around 75 and $225 \mathrm{MHz}$ which are the resonant frequencies of the dipoles. Fig. 9 illustrates that totalradiated power is equal to the sum of the self-radiated power of $a$ and $b$, and mutual-radiated power between coupled parts. 


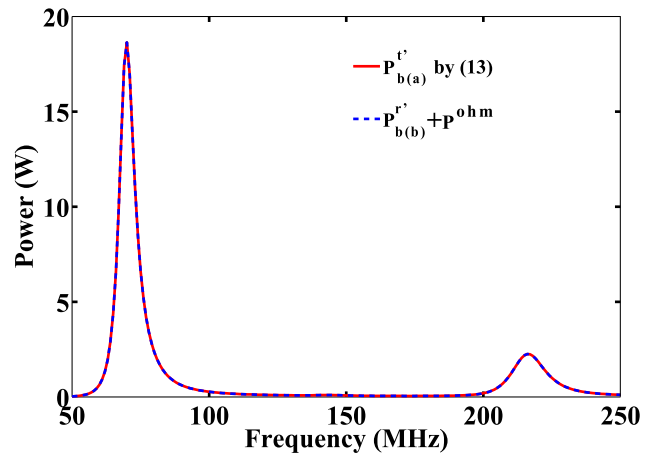

Fig. 8. Transferred power of coupled dipoles calculated by (13). $P_{b(a)}^{t^{\prime}}$ is the transferred power from $a$ to $b$.

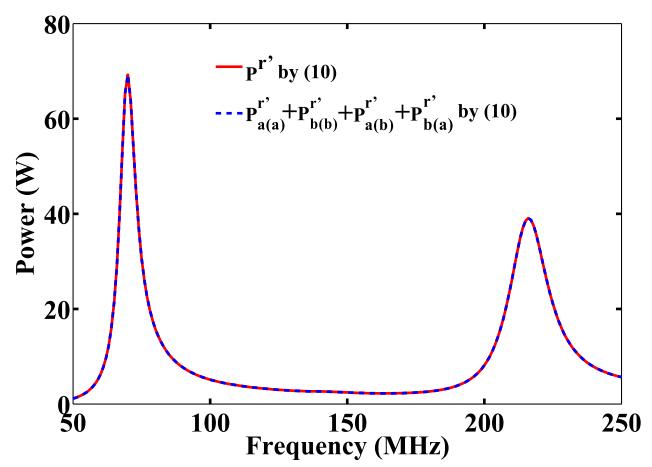

Fig. 9. Total-radiated power of two dipoles calculated by (10) compared with the summation of self- and mutual-radiated power between coupled parts.

We observe that the mutual power formulation is correct. And the radiated power results reach maximum at around 75 and 225 MHz.

\section{Two Square Loops}

The second example is a pair of two identical square loops parallel to each other. The length of each loop is $1 \mathrm{~m}$ and the width is $0.01 \mathrm{~m}$. The vertical distance between two loops is 0.1 $\mathrm{m}$. Loop $a$ has a $100-\mathrm{V}$ sinusoidal voltage source in the middle of one side. Loop $b$ is loaded with a $50-\Omega$ resistor. The self-radiated power is checked first. From Fig. 10, we can see that selfradiated power from (10) matches well with the result obtained with the PT. The radiated power results reach maximum at 75 and $150 \mathrm{MHz}$ which are the resonant frequencies of the loops. Fig. 11 illustrates that transferred power matches very well with power consumption on $b$, which means that the transferred power formulation derived from the energy conservation law is correct. And the transferred power results reach a maximum at 75 and $150 \mathrm{MHz}$ which are the resonant frequencies of the loops. Fig. 12 illustrates that total-radiated power is equal to the sum of the self- and mutual-radiated power between coupled parts, which means that the mutual power formulation is correct. Finally, the radiated power results reach maximum at 75 and $150 \mathrm{MHz}$.

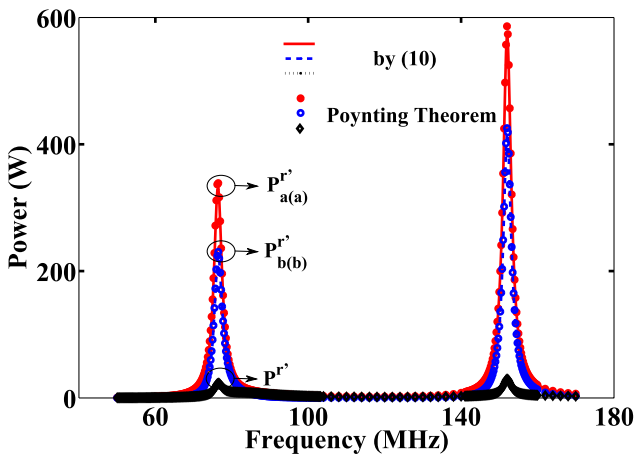

Fig. 10. Radiated power of two coupled loops calculated by (10) compared with the PT. $P^{r^{\prime}}$ is the total-radiated power of the system. $P_{a(a)}^{r^{\prime}}$ and $P_{b(b)}^{r^{\prime}}$ are self-radiated power by $a$ and $b$, respectively.

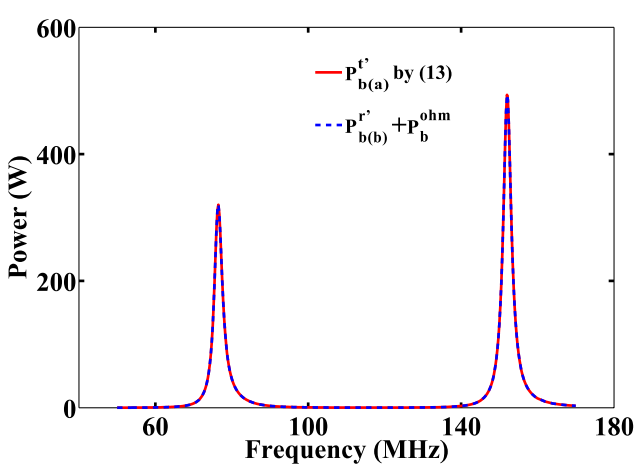

Fig. 11. Transferred power of coupled loops calculated by (13). $P_{b(a)}^{t^{\prime}}$ is the transferred power from $a$ to $b$.

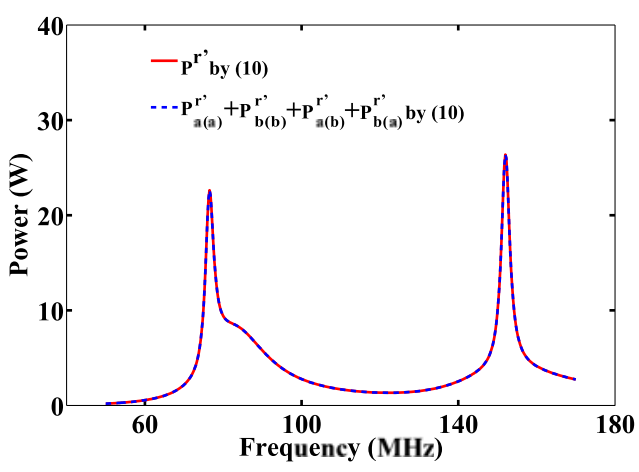

Fig. 12. Total-radiated power of two coupled loops calculated by (10) compared with the summation of self- and mutual-radiated power between coupled parts.

\section{Patch Antenna}

The third example is an air-filled patch antenna with its geometry depicted in Fig. 13. The patch is $1 \mathrm{~mm}$ above an infinitely large ground plane. The antenna has a line feed with a length of $7.5 \mathrm{~mm}$ and width of $1.6 \mathrm{~mm}$ attached to a $100-\mathrm{V}$ sinusoidal voltage source with a series resistance of $50 \Omega$. At a frequency of $10 \mathrm{GHz}, S_{11}$ reaches $-25 \mathrm{~dB}$. 


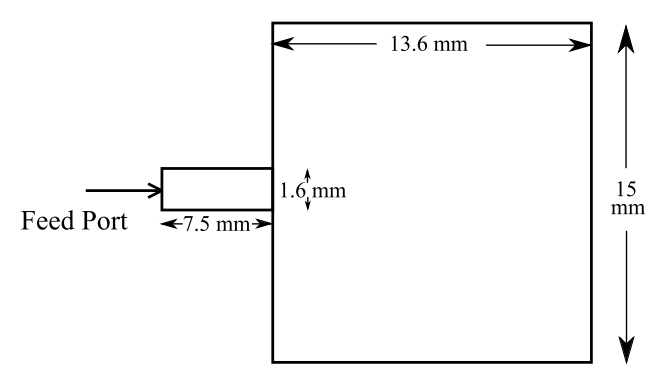

Fig. 13. Geometry of the patch.

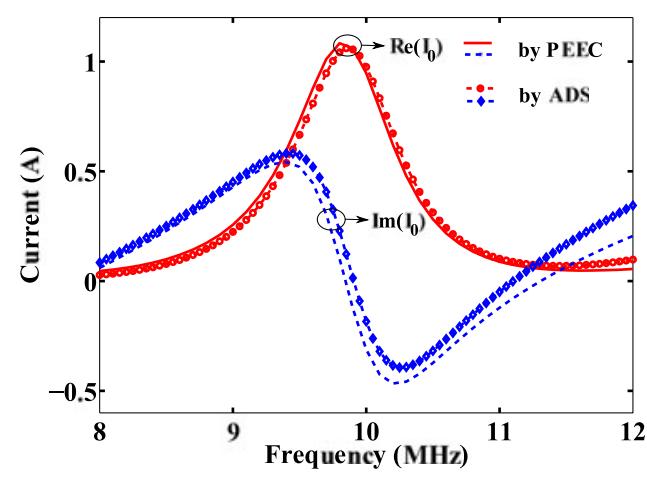

Fig. 14. Current through voltage source of the patch antenna calculated by PEEC compared with ADS results.

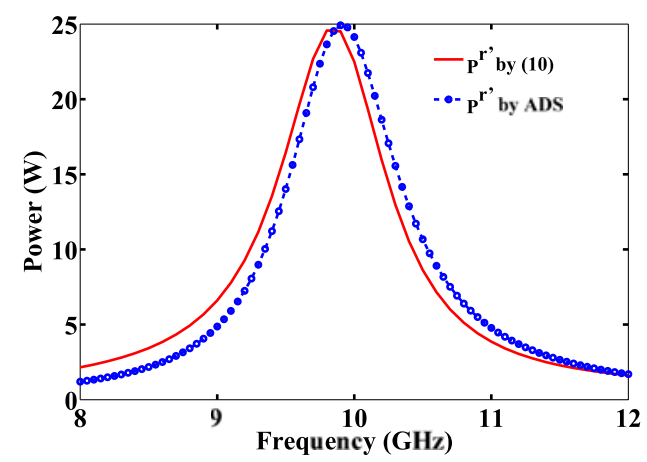

Fig. 15. Radiated power of the patch antenna calculated by PEEC compared with ADS results.

From Fig. 14, we observe that in the frequency range from 8 to $12 \mathrm{GHz}$, current flows through the voltage source matching well with ADS results.

Fig. 15 depicts that the radiated power changes with frequency and reaches maximum value at around $10 \mathrm{GHz}$. The results from (10) matches very well with ADS results.

In order to verify whether this system obeys the energy conservation law, we compare the radiated power with input power from the source in Fig. 16. Since the input power from the source is used in the patch for radiation, the two results should be equal to each other as is the case.

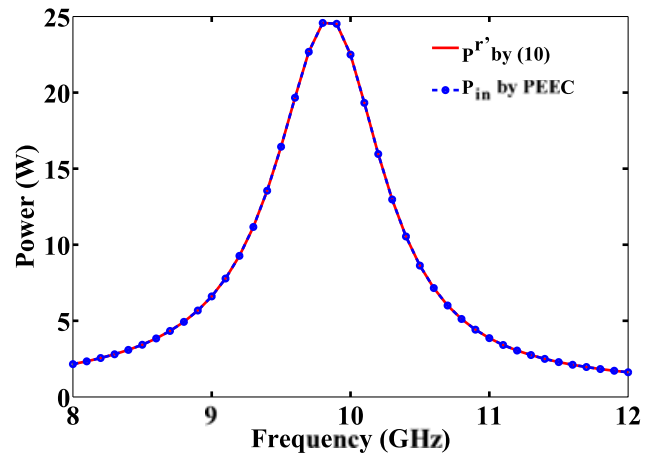

Fig. 16. Radiated power of the patch antenna calculated by PEEC compared with input power.

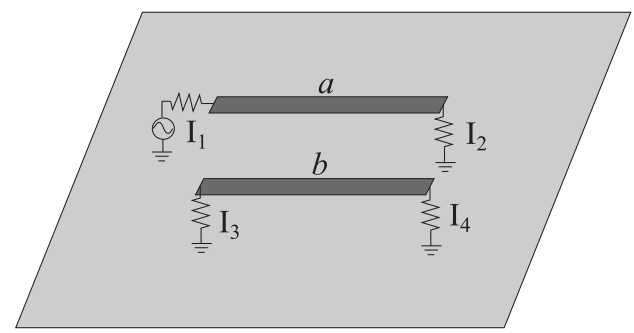

Fig. 17. Geometry of two coupled lines.

\section{E. Two Coupled Lines}

In this section, we discuss the power analysis on the PCB (see Fig. 17). The length of two coupled lines is $5 \mathrm{~cm}$, the width of the lines is $1 \mathrm{~mm}$ wide with a separation of $0.06 \mathrm{~mm}$. The coupled lines are air filled and are $0.1 \mathrm{~mm}$ above an infinitely large ground plane. The excitation is again a $100-\mathrm{V}$ sinusoidal voltage source.

1) Lower Frequency Case: All four termination resistors are all $50 \Omega$. The frequency range is from 1 to $4 \mathrm{GHz}$.

Fig. 18 shows the real and imaginary part of currents flow through four resistors in the four ends of coupled lines by PEEC and by ADS, respectively. And the currents match very well for these frequencies.

Fig. 19 depicts total-radiated power together with transferred power from $a$ to $b$. It is shown that for this frequency range, the radiated power is very small compared with transferred power. The transferred power from $a$ to $b, P_{a(b)}^{t^{\prime}}$ dominates in the total power analysis. Therefore, radiation can be neglected.

2) Higher Frequency Case: For very high frequencies, the radiated power is larger and cannot be neglectable. Four resistors are all $50 \Omega$. Frequency ranges from 10 to $14 \mathrm{GHz}$. Fig. 20 depicts real and imaginary part of currents flow through four resistors in the four ends of coupled lines by PEEC and by ADS, separately. The currents match well at relatively high frequencies with slight differences. The difference from ADS is due to the lack of a full-wave capability in the ADS solution. Fig. 21 depicts total-radiated power together with transferred power from $a$ to $b$. We can see clearly that at this frequency range, radiated power is even larger than transferred power at certain frequency points. Therefore, radiation cannot be neglected. 


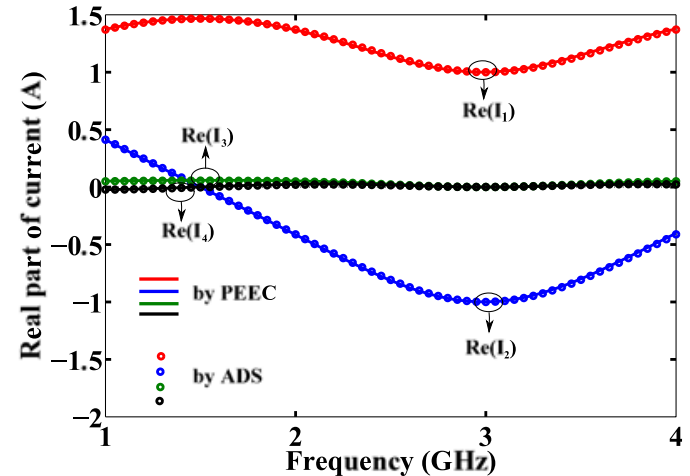

(a)

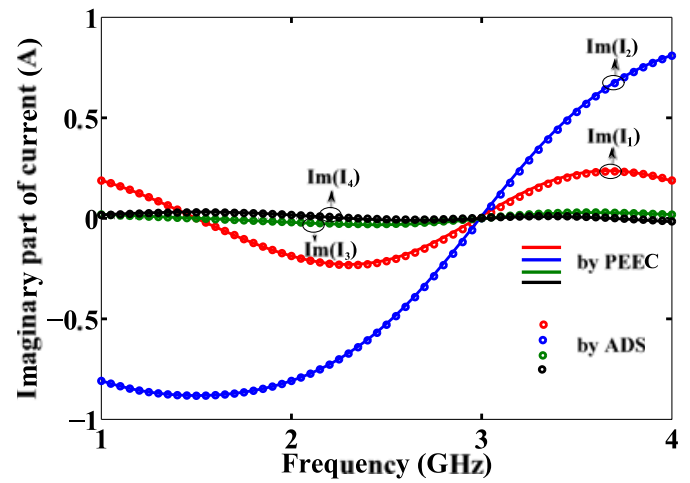

(b)

Fig. 18. (a) Real part of currents of coupled microstrip lines by PEEC in comparison with ADS results. (b) Imaginary part of currents coupled microstrip lines by PEEC in comparison with ADS results.

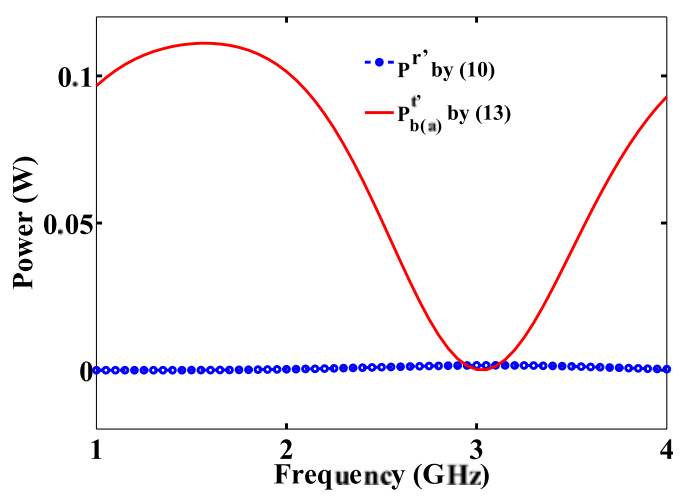

Fig. 19. Power analysis of coupled lines at lower frequency regime. $P_{r^{\prime}}$ is total-radiated power of the system calculated from (10), and $P_{b(a)}^{t^{\prime}}$ is transferred power from $a$ to $b$ evaluated in (13).

\section{CONCLUSION}

In this paper, a novel method for calculating and analyzing distributive radiation as well as the transfer characterization is proposed. The approach is based on the PEEC method and the energy conservation law. The approach allows the detailed characterization of the contribution of each circuit element to the transferred power and also the radiation. Examples are given for

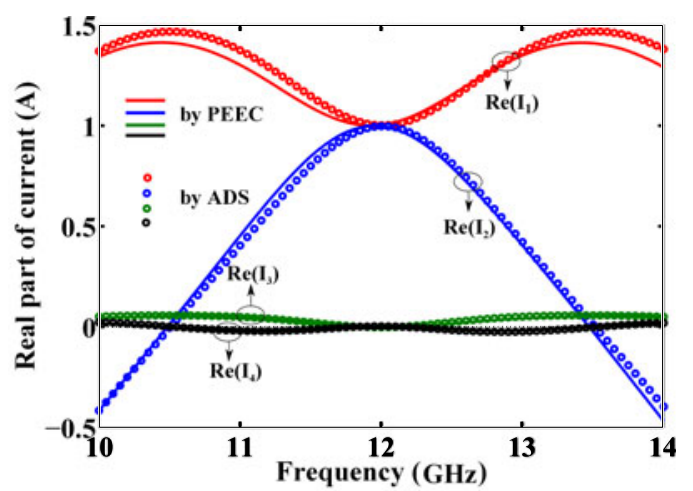

(a)

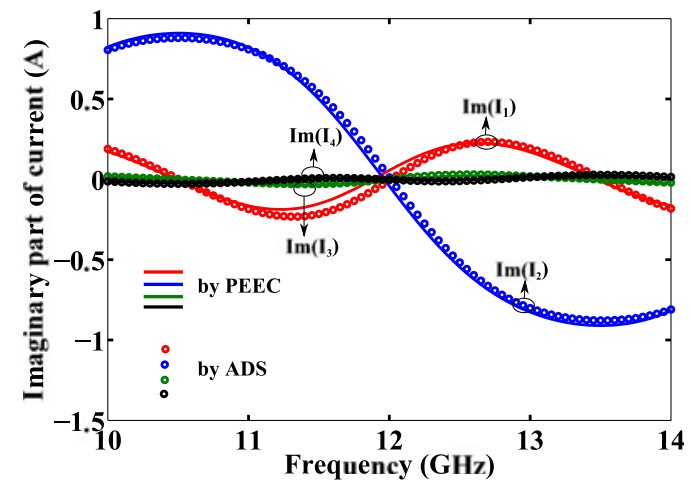

(b)

Fig. 20. (a) Real part of currents of coupled microstrip lines by PEEC in comparison with ADS results. (b) Imaginary part of currents coupled microstrip lines by PEEC in comparison with ADS results.

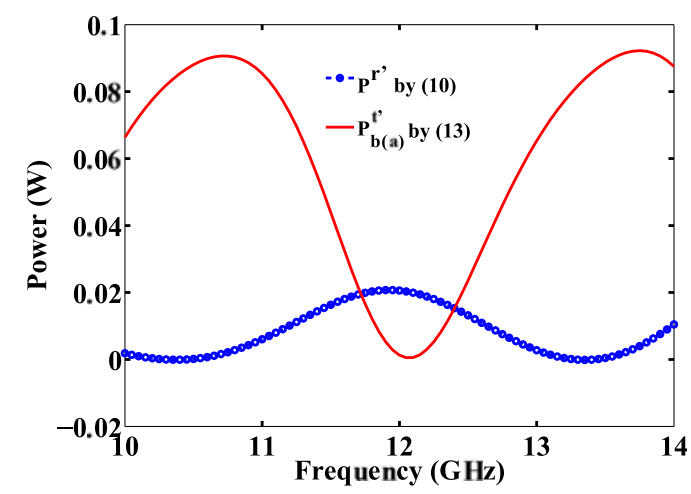

Fig. 21. Power analysis of coupled lines at lower frequency regime. $P_{r^{\prime}}$ is total-radiated power of the system calculated from (10), and $P_{b(a)}^{t^{\prime}}$ is transferred power from $a$ to $b$ by (13).

electric dipoles, magnetic dipoles, patch antennas and coupled microstrip lines. Further, the physical meanings of the power results are given. By properly utilizing the theory and the power distribution results, we are able to also give general guidelines. Examples are the wireless power transfer and the antenna design and optimization. 


\section{ACKNOWLEDGMENT}

The authors would like to thank reviewers and editors for the helpful comments, and W. C. Chew for the constructive suggestions.

\section{REFERENCES}

[1] A. Kurs, A. Karalis, R. Moffatt, J. D. Joannopoulos, P. Fisher, and M. Soljacic, "Wireless power transfer via strongly coupled magnetic resonances," Science, vol. 317, no. 5834, pp. 83-86, Jul. 2007.

[2] D. M. Sullivan, Electromagnetic Simulation Using the FDTD Method. New York, NY, USA: Wiley, 2000.

[3] J. Jin, The Finite Element Method in Electromagnetics. New York, NY, USA: Wiley, 1993.

[4] R. F. Harrington, Field Computation by Moment Methods. Piscataway, NJ, USA: IEEE Press, 1987.

[5] Z.-G. Qian, and W. C. Chew, "Fast full-wave surface integral equation solver for multiscale structure modeling," IEEE Trans. Antennas Propag., vol. 57, no. 11, pp. 268-272, Nov. 2009.

[6] A. E. Ruehli, "Equivalent circuit models for three dimensional multiconductor systems," IEEE Trans. Microw. Theory Tech., vol. MTT-22, no. 3, pp. 216-221, Mar. 1974.

[7] A. E. Ruehli, "Inductance calculations in a complex integrated circuit environment," IBM J. Res. Develop., vol. 16, no. 5, pp. 470-481, Sep. 1972.

[8] A. E. Ruehli, and P. A. Brennan, "Efficient capacitance calculations for three-dimensional multiconductor systems," IEEE Trans. Microw. Theory Tech., vol. 21, no. 2, pp. 76-82, Feb. 1973.

[9] A. E. Ruehli, G. Antonini, J. Esch, J. Ekman, A. Mayo, and A. Orlandi, "Non-orthogonal PEEC formulation for time and frequency domain EM and circuit modeling," IEEE Trans. Electromagn. Compat., vol. 45, no. 2, pp. 167-176, May 2003.

[10] G. Wollenberg and S. V. Kochetov, "Fast computation of radiated power distribution in coupled wire systems by the PEEC method," in Proc. IEEE Int. Symp. Electromagn. Compat., 2003, pp. 1152-1155.

[11] J. Nitsch, F. Gronwald, and G. Wollenberg, Radiating Non-Uniform Transmission Line Systems and Partial Element Equivalent Circuit Method. Hoboken, NJ, USA: Wiley, 2009.

[12] Y. S. Cao, L. J. Jiang, and A. E. Ruehli, "Distributive radiation characterization based on the PEEC method," in Proc. IEEE Int. Symp. Electromagn. Compat., 2014, pp. 203-208.

[13] B. Archambeault, S. Conner, M. S. Halligan, J. L. Drewniak, and A. E. Ruehli, "Electromagnetic radiation resulting from PCB/high-density connector interfaces," IEEE Trans. Electromagn. Compat., vol. 55, no. 4, pp. 614-623, Aug. 2013.

[14] L. K. Yeung, and K. Wu, "Generalized partial element equivalent circuit (PEEC) modeling with radiation effect," IEEE Trans. Microw. Theory Tech., vol. 59, no. 10, pp. 2377-2384, Oct. 2011.

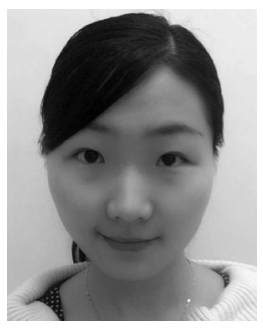

Ying S. Cao (S'14) received the B.S. degree in physics from the University of Science and Technology of China (USTC), Hefei, China, in 2012, and is currently working toward the Ph.D. degree at the University of Hong Kong, Hong Kong.

In 2011, she was a Visiting Student studying and researching on quantum teleportation at Clarendon Laboratory, University of Oxford. Since 2012, she has been with the Center of Electromagnetics and Optics, University of Hong Kong. Her research interests include computational electromagnetics, EMC/EMI,

and multiphysics modeling.

Ms. Cao won Excellent Graduate Award of USTC in 2012.

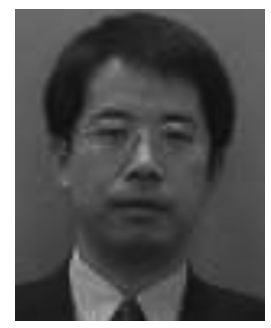

Li Jun Jiang (S'01-M'04-SM'13) received the B.S. degree in electrical engineering from the Beijing University of Aeronautics and Astronautics, Beijing, China, in 1993, the M.S. degree from the Tsinghua University, Beijing, in 1996, and the Ph.D. degree from the University of Illinois at Urbana-Champaign, Champaign, IL, USA, in 2004.

From 1996 to 1999, he was an Application Engineer with the Hewlett-Packard Company. Since 2004, he has been the Postdoctoral Researcher, the Research Staff Member, and the Senior Engineer at IBM T. J. Watson Research Center, Yorktown Heights, NY, USA. Since the end of 2009, he has been an Associate Professor with the Department of Electrical and Electronic Engineering, University of Hong Kong, Hong Kong. From September 2014 to March 2015, he has also been a Visiting Scholar at the University of California, Los Angeles, CA, USA. He is the Associate Editor of IEEE TRANSACTIONS ON ANTENNAS AND PROPAGATION, the Editor of Progress in Electromagnetics Research, the Associate Guest Editor of the PROCEEDINGS OF IEEE SPECIAL IssuE in 2011, 2012. He also serves as the Reviewer of IEEE transactions on several topics, and other primary electromagnetics and microwave-related journals. His research interests include electromagnetics, computational electromagnetics, IC signal/power integrity, IC EMC/EMI, antennas, multiphysics modeling, etc.

Dr. Jiang received the HP STAR Award in 1998. In 2003, he received the IEEE MTT Graduate Fellowship Award, and in 2004 the Y. T. Lo Outstanding Research Award. In 2008, he received the IBM Research Technical Achievement Award. He is an IEEE AP-S Member, an IEEE MTT-S Member, an ACES Member, and a Member of Chinese Computational Electromagnetics Society. He was the Semiconductor Research Cooperation Industrial Liaison for several academic projects. He was the TPC Member of IEEE Electrical Design for Advanced Packaging and Systems (EDAPS) since 2010, the TPC Member of 2013 IEEE International Conference on Microwave Technology and Computational Electromagnetics, the Scientific Committee Member of 2010 Workshop on Simulation and Modeling of Emerging Electronics, the Special Session Organizer of IEEE EDAPS, the International Review of Progress in Applied Computational Electromagnetics, Asia-Pacific Radio Science Conference, the Coorganizer of HKU Computational Science and Engineering Workshops in 2010-2012, the TC-9 and TC-10 Member of IEEE EMC-S since 2011, the TPC Chair of the Seventh International Conference on Nanophotonics, the TPC Member of the Third Conference on Advances in Optoelectronics and Micro/Nano Optics, the Cochair of International Workshop on Pulsed Electromagnetic Field at the Delft, The Netherlands, 2013, the Chair of Fourteenth IEEE Hong Kong AP/MTT Postgraduate Conference, and session chairs of many international conferences.

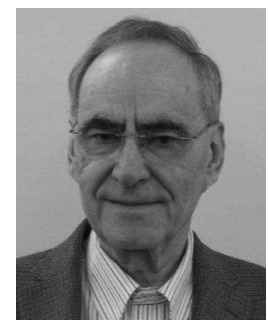

Albert E. Ruehli (LF'03) received the Ph.D. degree in electrical engineering in 1972 from the University of Vermont, Burlington, MA, USA, and an Honorary Doctorate in 2007 from the Lulea University, Lulea, Sweden.

He has been a member of various projects with IBM including mathematical analysis, semiconductor circuits and devices modeling, and as Manager of a VLSI Design and CAD Group. Since 1972, he has been at IBM's T. J. Watson Research Center, Yorktown Heights, NY, USA, where he was a Research Staff Member in the Electromagnetic Analysis Group. He is now an Emeritus of IBM Research and an Adjunct Professor in the EMC area at the Missouri University of Science and Technology, Rolla, MO, USA. He is the Editor of two books, Circuit Analysis, Simulation and Design (New York, NY, USA: North Holland, 1986, 1987) and he is an author or coauthor of more than 200 technical papers.

Dr. Ruehli has served in numerous capacities for the IEEE. In 1984 and 1985, he was the Technical and General Chairman, respectively, of the ICCD International Conference. He has been a Member of the IEEE ADCOM for the Circuit and System Society and an Associate Editor for the Transactions on Computer-Aided Design. He has given talks at universities including keynote addresses and tutorials at conferences, and has organized many sessions. $\mathrm{He}$ received IBM Research Division or IBM Outstanding Contribution Awards in 1975, 1978, 1982, 1995 and 2000. In 1982, he received the Guillemin-Cauer Prize Award for his study on waveform relaxation, and in 1999, he received a Golden Jubilee Medal, both from the IEEE CAS Society. In 2001, he received a Certificate of Achievement from the IEEE EMC Society for inductance concepts and the partial element equivalent circuit method. He received the 2005 Richard R Stoddart Award, and in 2007 he received the Honorary Life Member Award from the IEEE Electromagnetic Compatibility Society for outstanding technical performance. In 2010, he received a Best Paper Award at the EPEPS Conference for his study on optimized waveform relaxation. He is a Member of SIAM. 